\title{
An unusual intracerebral hemorrhage
}

\author{
Roya Alavi-Naini MD ${ }^{1}$, Ali Moghtaderi MD², Malyhe Metanat MD
}

\begin{abstract}
$\mathrm{A}^{20}$ 20-year-old man was admitted to hospital for fever and diarrhea. He was well until three days before admission when fever, nausea, vomiting and watery diarrhea suddenly occurred. The day before admission, he experienced epigastric pain and upper gastrointestinal bleeding. The patient was a student of veterinary medicine. There was no history of recent travel but he had exposure to cattle. On physical examination, he appeared ill and toxic with a temperature of $39.2^{\circ} \mathrm{C}$. The patient was tachycardic and tachypneic, and the conjunctivae were injected. On abdominal examination, he had epigastric tenderness without organomegaly. A diffuse maculopapular rash and petechiae were seen over the entire body, especially the chest and abdomen. Large echymoses were seen at the venipuncture sites on the ventral portion of the forearms. The results of laboratory tests are shown in Table 1 . Treatment was begun with oral ribavirin $(30 \mathrm{mg} / \mathrm{kg}$ loading dose, then $15 \mathrm{mg} / \mathrm{kg}$ every $6 \mathrm{~h}$ for four days followed by $7.5 \mathrm{mg} / \mathrm{kg}$ three times daily for six additional days) and intravenous ceftriaxone, along with platelet transfusions and supportive therapy.

Fever and petechiae were improved on the fifth day of admission but the following day the patient developed a headache, gait disorder, incontinence and a confusional state. On physical examination, speech was normal except for occasional hesitation but the patient was confused and demonstrated prominent attention and concentration defects. Neck stiffness was not noted. Cranial nerves and deep tendon reflexes were normal. Muscle tone was decreased only in the lower limbs where motor function was graded $3 / 5$ proximally
\end{abstract}

TABLE 1

Laboratory test results during hospital admission

\begin{tabular}{lcccc}
\hline & First day & Third day & Seventh day & 10th day \\
\hline Hematocrit (\%) & 33 & 25 & 24 & 27 \\
WBC/L & $5.4 \times 10^{9}$ & $2.5 \times 10^{9}$ & $4.5 \times 10^{9}$ & $6.7 \times 10^{9}$ \\
PMN (\%) & 75 & 60 & 63 & 65 \\
Platelets/L & $15 \times 10^{9}$ & $10 \times 10^{9}$ & $51 \times 10^{9}$ & $135 \times 10^{9}$ \\
AST (U/L) & 515 & 508 & 210 & 229 \\
ALT (U/L) & 91 & 160 & 226 & 247 \\
PT (s) & 24 & 16 & 15 & 12
\end{tabular}

ALT Alanine aminotransferase; AST Aspartate aminotransferase; PMN Polymorphonuclear; PT Prothrombin time; WBC White blood cells

and 4/5 distally. Plantar toe reflexes were extensor (ie, upward) bilaterally. The upper limbs were normal. Sensory examination could not be reliably performed due to the patient's confused state. The result of finger-to-nose testing was normal but standing and walking were abnormal. Notably, he could walk assisted with a wide-based stance but advanced with small, shuffling, hesitant steps. Turning was accomplished by a series of tiny, uncertain steps that were made by one foot, with the other foot being used as a pivot. A computed tomography (CT) scan of the brain was performed without contrast and a laboratory result was received that day which established the diagnosis. What did the CT of the brain show and what is the patient's diagnosis?

\footnotetext{
${ }^{1}$ Department of Infectious Diseases; and ${ }^{2}$ Neurology Department, Zahedan University of Medical Sciences, Zahedan, Iran

Correspondence and reprints: Dr Roya Alavi-Naini, Department of Infectious Diseases, Booali Hospital, Zahedan, 98531773, Iran.

Telephone 0098-541-3234551, fax 0098-541-3218848, e-mail ranaini@zdmu.ac.ir or ranaini@iranmedical.com
} 


\section{DIAGNOSIS}

CT scanning of the brain without contrast (Figure 1) showed large bilateral frontal parasagittal hematoma, worse on the right side. An immunoglobulin (Ig) M-ELISA serological test for Crimean-Congo Hemorrhagic Fever (CCHF) virus was reported positive on the fifth day of admission, and both IgM and IgG-ELISA tests were positive on the 10th day of admission. Two weeks later, the patient was improved. Another brain CT scan with contrast showed progressive resorption of the cerebral hematomas. The patient was discharged as improved on the 18th day of admission.

\section{DISCUSSION}

Viral hemorrhagic fevers have attracted the attention of the medical world for many reasons. Large outbreaks have affected populations in endemic areas, living mainly in inaccessible areas or refugee camps where living conditions are dramatically adverse. Medical facilities with scarce resources have played a role in amplifying transmission and infection control measures have been difficult or virtually impossible to establish (1). The widespread geographical distribution of the CCHF virus (more than 30 countries) and its ability to produce severe human disease with high mortality rates (up to 60\% of clinically apparent cases) make CCHF a major public health concern (2). Despite this high lethality rate, up to $80 \%$ of the infections may be subclinical.

CCHF can produce a severe hemorrhagic fever with shock, disseminated intravascular coagulation (DIC), frequent extensive bleeding and severe thrombocytopenia. It is caused by a recently emerged bunyavirus. Following an incubation period
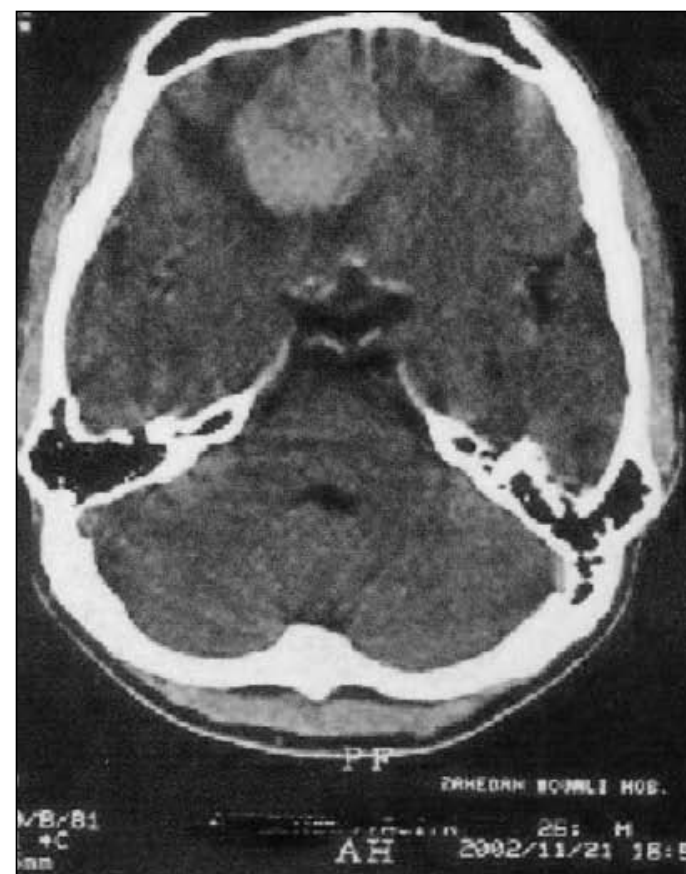

Bhisonk nOMAI HOS

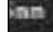

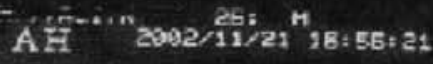

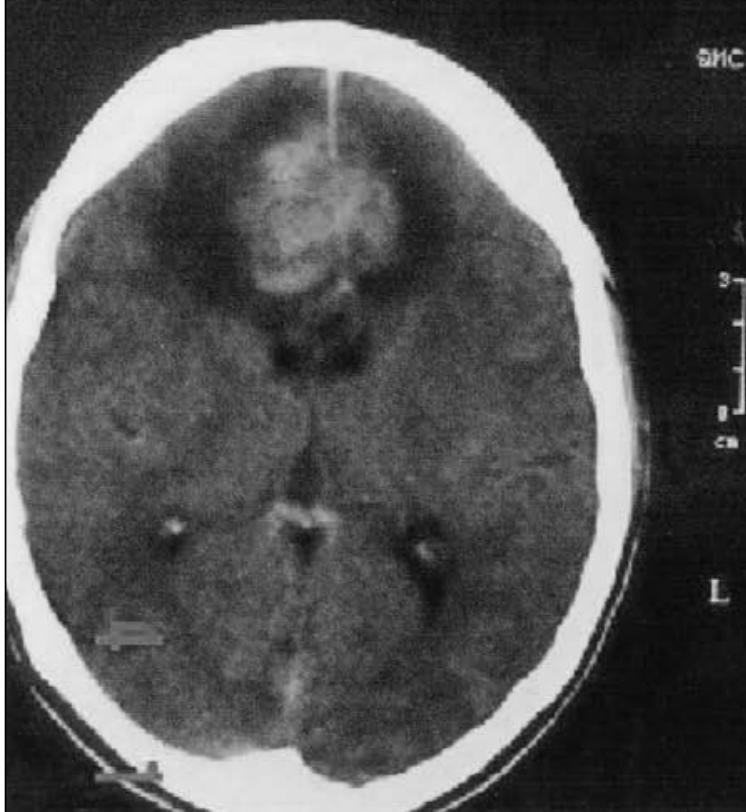

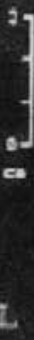

L.

ces

He

L

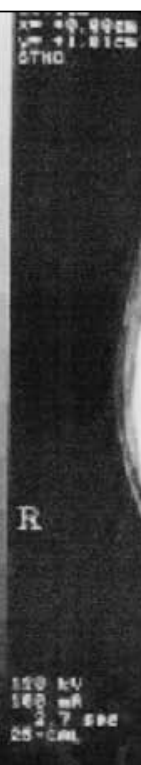

W150 $4 L+90$
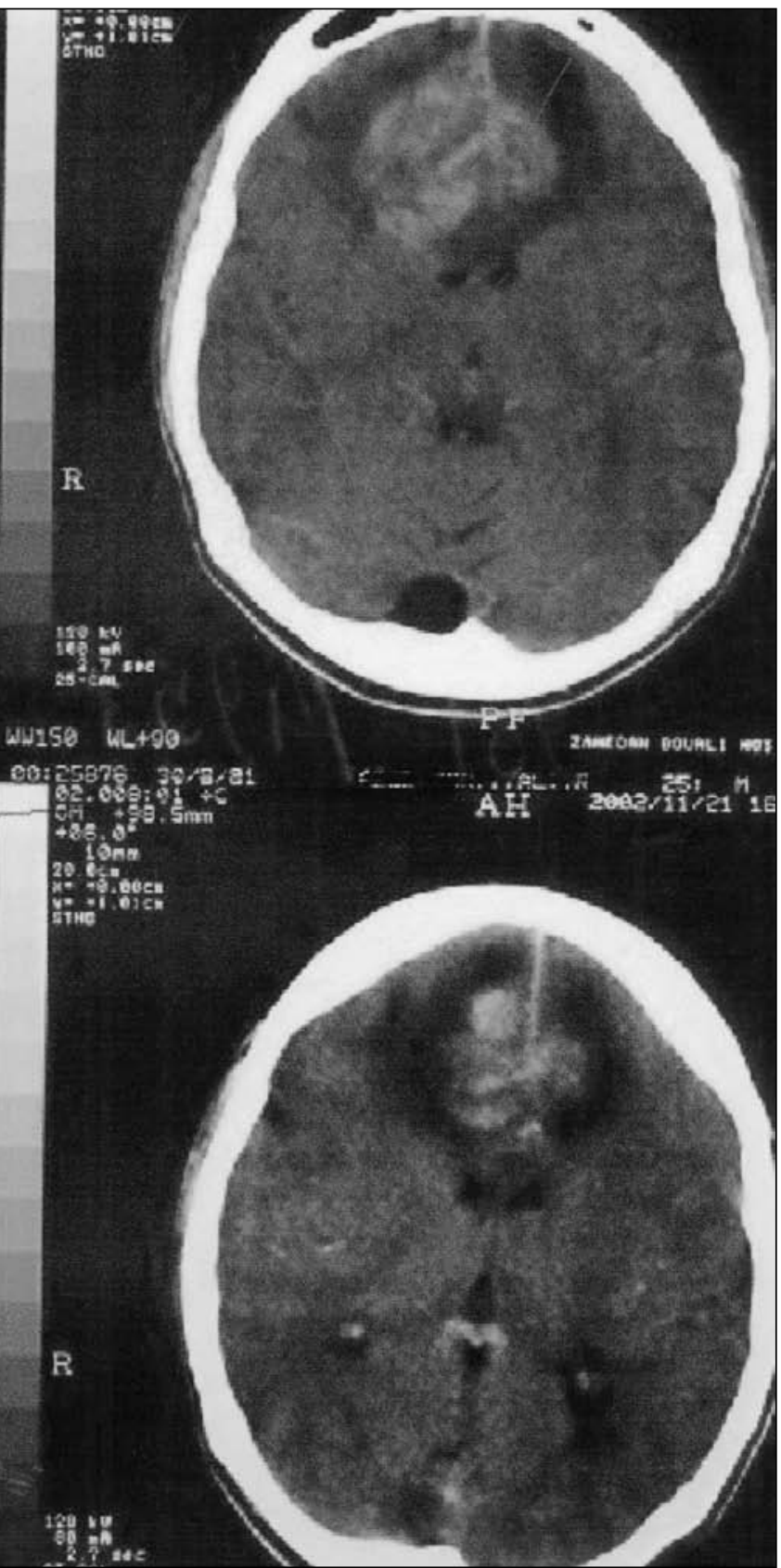

Figure 1) Computed tomography scan of the head, showing large bilateral frontal parasagittal hematoma 
of two to nine days, patients have a sudden onset of fever, nausea, severe headache and myalgia. Petechial rash and hemorrhagic signs such as hematemesis and melena supervene on days three to six of the illness. Other sites of bleeding may occur, and in 1985 Swanepoel (3) reported a fatal case of $\mathrm{CCHF}$ when the patient died of complications following surgical therapy of a cerebral hemorrhage. Death from CCHF usually occurs on days five to 14 of illness. Patients with fatal infections have thrombocytopenia and markedly elevated levels of liver enzymes. Total protein, albumin, fibrinogen and hemoglobin levels are decreased. The coagulation process is grossly disturbed, with findings that indicate the occurrence of DIC. Clinical and pathological changes which are evident at an early stage of the disease carry a high probability of a fatal outcome, while less marked changes are seen in nonfatal infections (4). Significant risk factors for disease include exposure to animal blood or tissue in abattoirs, as well as a history of tick bites (5). Seropositivity rates are similar in both sexes and increase significantly with age among nomadic persons. Activities related to herding activities (ie, sleeping outside during seasonal migrations and having contact with sick animals) also seem to be risk factors for infection (6). Diagnosis of infection may be based on detection of CCHF IgG by ELISA, which possesses high sensitivity and specificity (7). As well, acute infection may be detected early by either an IgM-capture ELISA assay, an antigen-detection ELISA (8) or by reverse transcription-polymerase chain reaction (2,9). In 1995, Fisher-Hoch et al (10) reported for the first time that three health workers in Pakistan were successfully treated with oral ribavirin.

During the past three years (2000 to 2002 inclusive), there was an epidemic of CCHF in southeast Iran, possibly due to an increase in the import of infected cattle and sheep from other countries. Of 161 patients with clinically suspected acute disease, 107 had acute CCHF proven by positive IgG- and IgMELISA serological tests (5). Only five patients in the confirmed group died but the overall mortality may have been underestimated due to the fact that serological tests before death were not possible on all 161 patients, and the nontested patients were more likely to have died from their illness. Although severe thrombocytopenia is the main cause of cerebral hemorrhage in our patient, we believe that this is the first patient ever described with parasagittal intracerebral hematomas and gait disorder secondary to CCHF. Frontal lobe disorder of gait is induced by parasagittally located cerebral lesions and their connection with the basal ganglia, such as that which occurs with cerebral hematomas. The disorder is sometimes called frontal lobe ataxia or gait apraxia because the difficulty of walking cannot be accounted for by weakness or cerebellar incoordination. The use of corticosteroids and the natural progressive resorption of blood reversed the clinical syndrome of gait disorder in our patient. In conclusion, any development of central nervous system-related symptoms in a CCHF patient during the acute phase must be considered an emergency and related to hemorrhage, until proven otherwise. Symptoms of intracerebral hemorrhage may be obvious but may also manifest in subtle and unusual ways, as in our patient with gait apraxia.

ACKNOWLEDGEMENTS: We thank Dr Rahimi-Movaghar and Dr Heshmatipoor for critically reviewing the manuscript.

\section{REFERENCES}

1. Crowcroft NS, Morgan D, Brown D. Viral haemorrhagic fevers in Europe - effective control requires a co-ordinated response. Euro Surveill 2002;7:31-2.

2. Flick R, Flick K, Feldmann H, Elgh F. Reverse genetics for crimeancongo hemorrhagic fever virus. J Virol 2003;77:5997-6006.

3. Swanepoel R, Shepherd AJ, Leman PA, Shepherd SP, Miller GB. A common-source outbreak of Crimean-Congo haemorrhagic fever on a dairy farm. S Afr Med J 1985;68:635-7.

4. Swanepoel R, Gill DE, Shepherd AJ, Leman PA, Mynhardt JH, Harvey S. The clinical pathology of Crimean-Congo hemorrhagic fever. Rev Infect Dis 1989;11(Suppl 4):S794-800.

5. Ministry of Health and Treatment, Centers for Disease Control and Prevention, Internal Bull. Crimean-Congo Hemorrhagic Fever. Zahedan: Zahedan University School of Medical Sciences, 2003.

6. Chapman LE, Wilson ML, Hall DB, et al. Risk factors for CrimeanCongo hemorrhagic fever in rural northern Senegal. J Infect Dis 1991;164:686-92.

7. Saijo M, Qing T, Niikura M, et al. Recombinant nucleoprotein-based enzyme-linked immunosorbent assay for detection of immunoglobulin $\mathrm{G}$ antibodies to Crimean-Congo hemorrhagic fever virus. J Clin Microbiol 2002;40:1587-91.

8. Saluzzo JF, Le Guenno B. Rapid diagnosis of human Crimean-Congo hemorrhagic fever and detection of the virus in naturally infected ticks. J Clin Microbiol 1987;25:922-4.

9. Burt FJ, Leman PA, Smith JF, Swanepoel R. The use of a reverse transcription-polymerase chain reaction for the detection of viral nucleic acid in the diagnosis of Crimean-Congo haemorrhagic fever. J Virol Methods 1998;70:129-37.

10. Fisher-Hoch SP, Khan JA, Rehman S, Mirza S, Khurshid M, McCormick JB. Crimean Congo-haemorrhagic fever treated with oral ribavirin. Lancet 1995;346:472-5. 


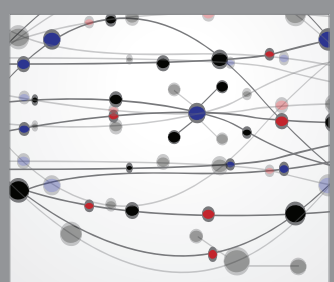

The Scientific World Journal
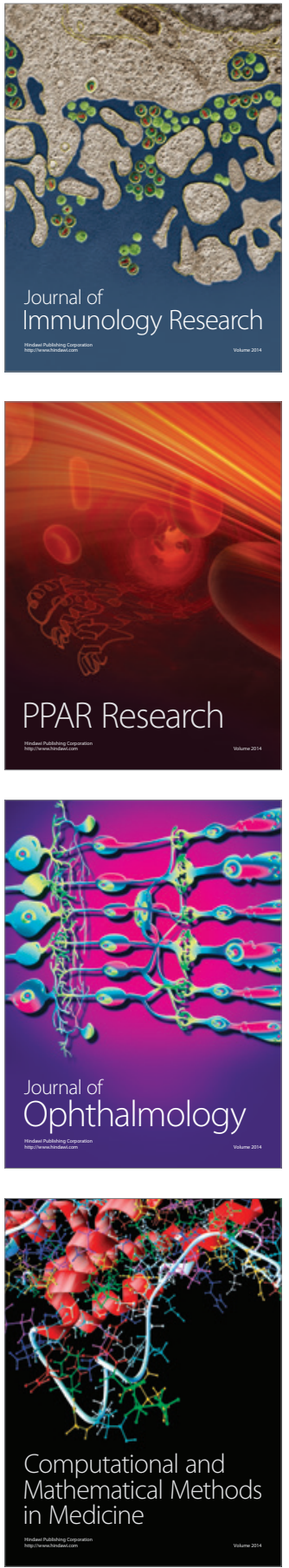

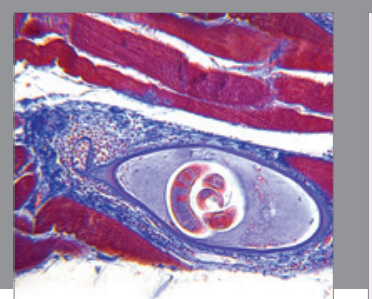

Gastroenterology Research and Practice

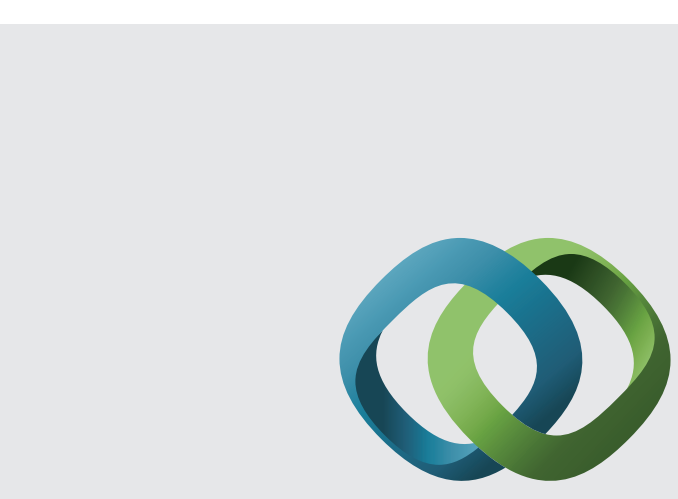

\section{Hindawi}

Submit your manuscripts at

http://www.hindawi.com
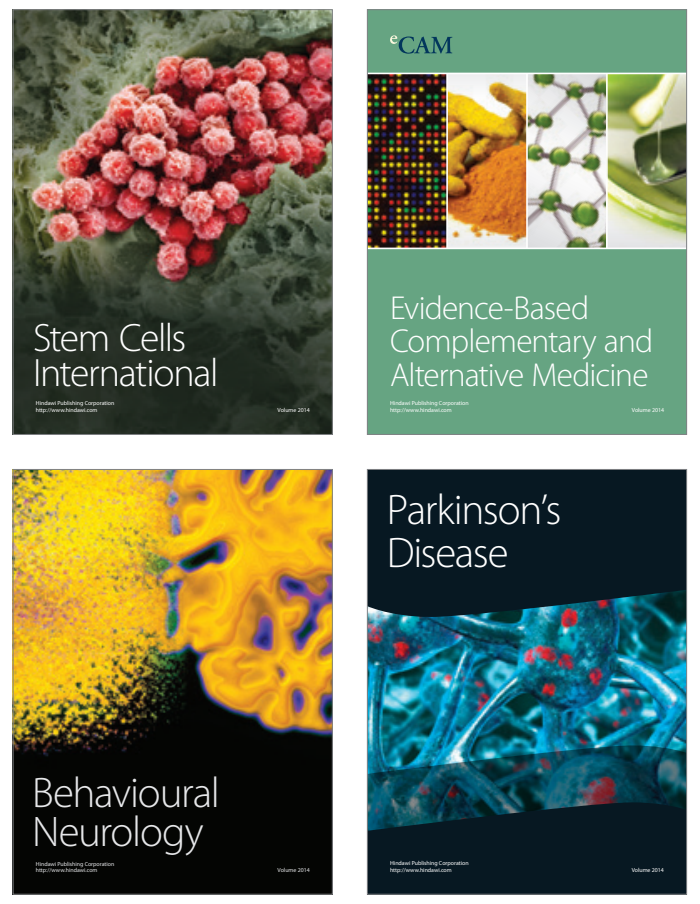
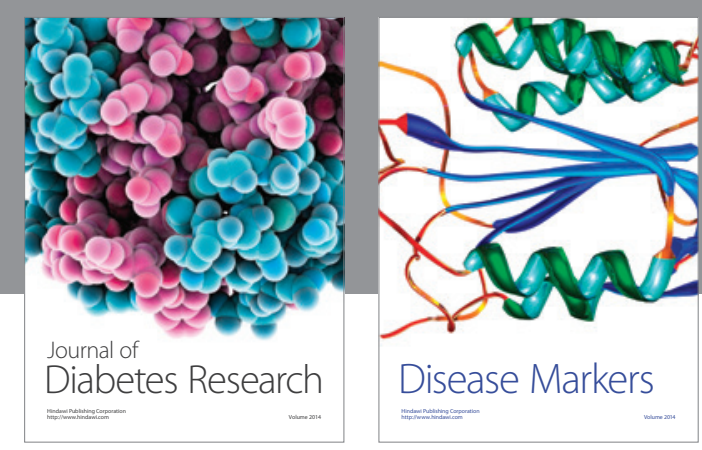

Disease Markers
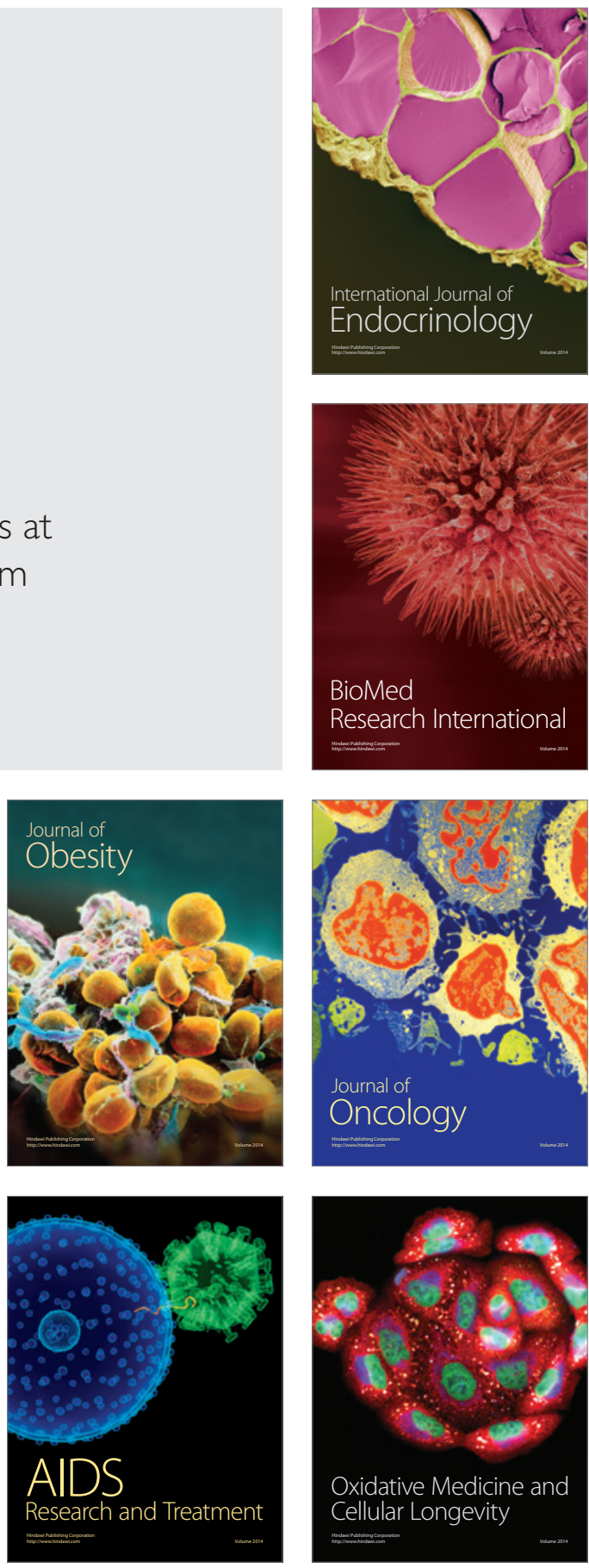